Polycythemia prevalence with intranasal vs. intramuscular testosterone

\title{
A cross-sectional comparison of secondary polycythemia in testosterone-deficient men treated with nasal testosterone gel vs. intramuscular testosterone cypionate
}

Jordan C. Best; Daniel Gonzalez; Thomas A. Masterson; Ruben Blachman-Braun;

Raghav Pai; Ranjith Ramasamy

${ }^{1}$ Department of Urology, Miller School of Medicine, University of Miami, Miami, FL, United States

Cite as: Can Urol Assoc J 2020 July 27; Epub ahead of print. http://dx.doi.org/10.5489/cuaj.6651

Published online July 27, 2020

$* * *$

\section{Abstract}

Introduction: Secondary polycythemia is a known adverse effect of testosterone replacement therapy (TRT). Different testosterone formulations are available, with significantly different half-lives, which have varying influences on the development of secondary polycythemia. Herein, we compared the prevalence of secondary polycythemia in testosterone-deficient men treated with intranasal testosterone gel (Natesto) vs. intramuscular testosterone cypionate (TC) therapy.

Methods: We performed a cross-sectional analysis of secondary polycythemia (hematocrit [Hct] $\geq 54 \%$ ) in men who received TRT. We included a total of 60 men: 30 men who received Natesto (4.5\% testosterone gel [tid, $5.5 \mathrm{mg} /$ nostril, $11 \mathrm{mg} / \mathrm{dose}, 33 \mathrm{mg} /$ day]), and 30 who received TC (between 0.5 and $1.0 \mathrm{~mL}$ or $100-200 \mathrm{mg}$ intramuscularly weekly). A univariable and multiple regression analysis was performed considering last Hct measurement as the main outcome. The analyzed variables included were age, body mass index (BMI), smoking history, treatment group, and testosterone levels on followup.

Results: We identified polycythemia (Hct $\geq 54 \%$ ) in $10 \%(3 / 30)$ of men who received TC. Additionally, in men treated with TC, 33.3\% (10/30) had a Hct $\geq 50 \%$ during therapy. None of the men who received Natesto had a Hct $\geq 50 \%$ during therapy. On multivariable linear regression analysis, we demonstrated that the use of TC increased Hct by $3.24 \%(95 \%$ confidence interval $[\mathrm{CI}] 0.74-5.73 \%$; $\mathrm{p}=0.012$ ) compared to Natesto.

Conclusions: The prevalence of polycythemia in men treated with Natesto was markedly lower compared to the men who received TC therapy. 


\section{Introduction}

The prevalence of middle-aged males diagnosed with testosterone deficiency is estimated to be 6.5 million by 2025 , with an approximate $0.8 \%$ to $1.6 \%$ yearly decline in total testosterone after age $30 .{ }^{1}$ The most common treatment for males with testosterone deficiency is testosterone replacement therapy (TRT). In males 18-45 years old, testosterone prescription sales have quadrupled in the past decade. ${ }^{2}$ In addition to ameliorating testosterone deficiency symptoms, TRT can lead to increased muscle mass, bone mineral density and improve insulin resistance. ${ }^{3,4}$ However, TRT has several common side effects, including increased in estradiol, acne, gynecomastia, and erythrocytosis. ${ }^{5-7}$ Commonly used testosterone (T) formulations include IM injectables, transdermal gels, and subcutaneous pellets. While all TRT preparations are effective, the likelihood of the associated side effects are determined by method of administration, pharmacokinetics, and dosage. ${ }^{8}$ Initially described by Dobs et al., several studies examined the incidence of erythrocytosis associated with IM injections, in particular testosterone cypionate (TC) and enanthate, over transdermal formulations have the highest incidence of erythrocytosis, almost approaching $40 \%{ }^{9}$

The erythrogenic effect of TRT has been recognized since the 1960s, however, the different mechanisms proposed to explain this observation remain poorly understood; including increased erythropoietin production by the kidneys, ${ }^{10}$ increase of erythroid progenitor cells in bone marrow, ${ }^{11}$ and suppression of hepcidin leading to increased iron absorption and subsequent increase in systemic iron transport and erythropoiesis. ${ }^{12}$ Bachman et al., showed that supraphysiologic $\mathrm{T}$ levels suppressed hepcidin in a dose- and age dependent manner by more than $50 \%$, and resulted with an increased hematocrit along with stable erythropoietin levels. Since the release of a new warning label in 2014 by the FDA, thrombotic and cardiovascular risks of testosterone therapy have come under increased scrutiny. ${ }^{13}$

We conducted a clinical trial with Natesto - intransal testosterone gel, and demonstrated that the majority of men were able to maintain their gross semen parameters during treatment. ${ }^{14}$ Natesto has a different pharmacokinetic profile and much shorter half-life compared to TC. ${ }^{15,16}$ It is believed that semen parameters were maintained during treatment with Natesto likely due to the short half-life and frequent troughs in serum $\mathrm{T}$ throughout the day and throughout the night, which allow for secretion of GnRH. ${ }^{14}$ Due to this this novel finding and unique pharmacokinetic profile, we hypothesized that the short half-life of Natesto (30 minutes) would contribute to lower prevalence of polycythemia as compared to the long half-life of TC (6.9 days). ${ }^{17}$ Our objective was to perform a cross-sectional analysis to evaluate the prevalence of secondary polycythemia for men on either Natesto or TC, and prospectively evaluate the changes in $\mathrm{T}$ and Hct after treatment. 
Polycythemia prevalence with intranasal vs. intramuscular testosterone

\section{Methods}

\section{Patient identification and data acquisition}

We performed a cross-sectional analysis of Hct levels in men being treated for testosterone deficiency with either Natesto or TC. A total of 118 men were identified who received either Natesto or TC between May 2017 and November 2019. Furthermore, we analyzed the testosterone levels at the time of the highest Hct or last recorded Hct on follow-up after at least 3 months of therapy. After removing patients with missing data and/or lab work, 60 men remained: 30 who received Natesto and 30 who received TC. Men were diagnosed with testosterone deficiency according to AUA guidelines, which require both clinical symptoms of testosterone deficiency (i.e. decreased libido, energy, and erectile dysfunction), as assessed by history during their initial clinic encounter, in addition to biochemical evidence of low serum $\mathrm{T}(\leq 300 \mathrm{ng} / \mathrm{dL})$. All men had their labs drawn from the same lab between 7:00 am - 10:00 am. Men who were interested in preserving fertility were prescribed Natesto and men not interested in fertility were prescribed TC. Men receiving Natesto administered the drug three times daily based on manufacturer's recommendations (Natesto 4.5\% testosterone gel [t.i.d., $5.5 \mathrm{mg} / \mathrm{nostril}, 11 \mathrm{mg}$ / dose, 33mg/day]), manufactured by Haupt Pharma (Regensburg, Germany). Men on injectable TC formulations injected between 0.5 and $1.0 \mathrm{~mL}$ or $100-200 \mathrm{mg}$ intramuscularly weekly.

We performed a cross-sectional analysis to evaluate the overall prevalence of secondary polycythemia during treatment with Natesto and TC. Since the definition of erythrocytosis in the literature ranges from $50-54 \%$, we defined erythrocytosis as Hct $\geq 54 \%$, on follow-up based on the recommendations by the Endocrine Society Guidelines, ${ }^{18}$ which is the cutoff utilized in clinical practice to identify patients requiring cessation of treatment and/or phlebotomy. For a secondary endpoint, we assessed the number of men with a Hct above the other cutoffs defined by the Endocrine Society Guidelines (Hct $>50 \%$, Hct $>52 \%$ ) to help guide clinical decision making. A post-hoc power analysis with $n=60$ (30 in each group) looking at incidence of HCT $>50 \%$ and an alpha of 0.05 would result in a power of $95 \%$. This study was submitted to and approved by the institutional review board of the University of Miami, Miller School of Medicine, Miami, FL, USA.

\section{Statistical analysis}

All Statistical Analysis was performed using Statistical Package for the Social Sciences (SPSS) Version 24 with $\mathrm{p}<0.05$ considered statistically significant. Categorical variables were presented as absolute values and frequencies and were analyzed with a Chi-square or Fisher's exact test as required. Continuous variables were reported as the mean \pm standard deviation and the Student $\mathrm{T}$ test was utilized to compare groups. A univariable and multivariable regression analysis was performed considering last Hct measurement as the main outcome. The analyzed variables included were age, BMI, smoking history, treatment group and testosterone levels on follow-up. To avoid multicollinearity, before running a multivariable regression analysis, we confirmed that none of the variables had a high degree of correlation $(r> \pm 0.7)$ and calculated the tolerance of 
each independent variable. Additionally, we calculated the Cook's distance of each individual patient to determine the influence of data points and performed the Durbin-Watson test to assess for autocorrelation in the residuals.

\section{Results}

The clinical and demographic characteristics of the analyzed men are evident in Table 1 . The men treated with $\mathrm{TC}$ were older $(\mathrm{TC}=54.9 \pm 11.8$ years, vs. Natesto $=36.6 \pm 7.6$ years $)$ than men treated with Natesto. Men on TC had a longer mean treatment duration than men treated with Natesto ( $6.9 \pm 4.3$ vs. $4.7 \pm 1.5$ months). Smoking history was present in $8(26.7 \%)$ of men treated with TC and in $6(20 \%)$ of men treated with Natesto $(\mathrm{p}=0.542)$. There were no significant differences in baseline serum testosterone or Hct between the two groups $(p>0.05)$ (Table 1).

Polycythemia (Hct $\geq 54 \%$ ) prevalence in men on TC was $10 \%(3 / 30)$ vs $0 \%(0 / 30)$ for men on Natesto. Additionally, for patients on TC, 33.3\% (10/30) had a Hct $\geq 50 \%$ during therapy compared to Natesto which had no patients with a Hct $\geq 50 \%$ during therapy (Figure 1). On univariable linear regression, age and treatment group significantly contribute to hematocrit on follow-up. In the multivariable regression analysis model, there was independence of residuals (Durbin-Watson $=1.999)$. There was no evidence of multicollinearity, as evaluated in the independent variables with no results of tolerance less than 0.1 , and there was no case with a Cook's distance above 1 . The multivariable regression model significantly predicted follow-up $\operatorname{Hct}[F(5,54)=2.822, \mathrm{p}=0.025]$, and accounts for a variation in hematocrit of $13.4 \%\left(\mathrm{R}^{2}=\right.$ 0.207 , adj. $\left.\mathrm{R}^{2}=0.134\right)$. After adjusting for covariables, the treatment group significantly contributed to the model, showing that using TC increase Hct by 3.24\% (95\% CI: $0.74 \%$ $5.73 \%, \mathrm{p}=0.012$ ) when compare to Natesto (Table 2).

\section{Discussion}

To the best of our knowledge, this is the only study comparing the prevalence of secondary polycythemia in testosterone deficient patients treated with either intranasal testosterone (Natesto) or IM TC therapy. There was a lower prevalence of secondary polycythemia (Hct $\geq 54 \%)$ in the cohort treated with Natesto ( $0 \%$ ) compared to that on TC therapy $(10 \%)$. In addition, using various cutoffs for Hct, significant differences were seen between treatment groups. In men treated with TC, $33.3 \%$ (10/30) had a Hct $\geq 50 \%$ and $20 \%(6 / 30)$ had a Hct $\geq 52 \%$ on follow-up, compared to men treated with Natesto, which had no one develop a Hct $\geq 50 \%$ during treatment. The prevalence of secondary polycythemia due to TC is comparable to previous studies that have evaluated this phenomenon. ${ }^{19}$

A study performed by Bachman et al., showed that supraphysiologic $\mathrm{T}$ levels suppressed hepcidin in a dose- and age dependent manner by $>50 \%$ and resulted with an increased hematocrit along with stable erythropoietin levels. His study also supported the hypothesis that older men respond to testosterone with a relatively greater level of hepcidin suppression than younger men. This mechanism may be responsible for the difference in prevalence of 
polycythemia and change in Hct seen in TC patients, as the average age was 17.4 years older in our cohort. Since men interested in fertility preservation were treated with Natesto, this group was younger that the TC group, who were not interested in fertility preservation. Additionally, post-treatment levels of testosterone were significantly higher than Natesto $(983.1 \pm 394.1 \mathrm{ng} / \mathrm{dL}$ vs $640.3 \pm 302.4 \mathrm{ng} / \mathrm{dL}$ ), and this could have contributed to the observed difference. However, in an attempt to further analyze the effect of different independent variables on follow-up Hct, we performed a multivariable analysis that was adjusted by variables including age, BMI, smoking history and testosterone on follow-up. Our findings indicate that the most influential factor contributing to an increase in Hct on follow-up was the use of TC.

Previous studies have demonstrated that different $\mathrm{T}$ formulations may have varying effects on erythropoiesis, and some have proposed that the troughs in serum $\mathrm{T}$ between doses are most predictive of developing polycythemia ${ }^{20,21}$ Importantly, the pharmacokinetic profile and TID dosing of Natesto allows T levels to return to baseline multiple times daily, whereas TC leads to steady state levels over days. This property of Natesto was highlighted in our published clinical trial, in which the majority of men on Natesto maintained semen parameters during treatment. This was likely attributed to the maintenance of pulsatile secretion of GnRH that allowed daily release of gonadotropins ${ }^{22}$ Regardless of the proposed mechanisms for the erythrogenic effect of $\mathrm{T},{ }^{10-12}$ Natesto's short half-life and frequent troughs in $\mathrm{T}$ during treatment would have less of an influence on these mechanisms. Of note, although Natesto can provide symptom relief similar to that of TC, the TID administration that Natesto requires can be cumbersome for patients when compared to a single injection that lasts for weeks. ${ }^{22}$

The difference in the hematologic effect from these different formulations can have substantial clinical implications. The Framingham study, a 34-year follow up study evaluated the risk between cardiovascular disease (CVD) mortality and HCT, and found HCT to be an important risk factor for CVD mortality, including congestive heart disease, myocardial infarction, angina pectoris and stroke. ${ }^{23}$ According to the Endocrine Society clinical practice guidelines, a Hct $>50 \%$ should be a contraindication for initiation of $\mathrm{T}$ therapy, and a Hct $>54 \%$ should warrant discontinuation of the medication..$^{18} \mathrm{~A}$ previous systematic review and metaanalysis, which included 51 studies, found that an increase in Hct was the most frequent adverse effect experienced by men on T therapy (WMD, 3.18\%; 95\% CI, 1.35 to 5.01 ). ${ }^{24}$ Given the frequency of secondary polycythemia seen in men on TRT and the guidelines recommended by the Endocrine Society, having an option of TRT that minimizes the risk of polycythemia is optimal for clinical practice.

Our study had several limitations, including its retrospective nature, variability in dosages of TC taken and although adequately powered, a relatively small sample size and a non-age matched cohort. Strengths of the study are the novelty in the literature, adequate power, directly comparing the biochemical effects of TC and short-acting Natesto, multivariable regression analysis demonstrating mode of therapy as an independent predictor of polycythemia. Further 
prospective randomized trials are needed, in which men are matched by age, serum testosterone and hematocrit levels on baseline to confirm our findings.

\section{Conclusions}

The prevalence of polycythemia in men treated with Natesto were markedly lower compared to the men who received testosterone cypionate injections. Given that the most frequent adverse effect of TRT is the resulting polycythemia, Natesto could be a potential option if this is a concern, although larger prospective studies with age-matched cohorts are needed to validate the finding in our cross-sectional study. 


\section{References}

1. Araujo AB, Esche GR, Kupelian V, et al. Prevalence of symptomatic androgen deficiency in men. J Clin Endocrinol Metab. 2007;92:4241-7.

2. Rao PK, Boulet SL, Mehta A, et al. Trends in testosterone replacement therapy use from 2003 to 2013 among reproductive-age men in the United States. The Journal of urology. 2017;197:1121-6.

3. Kapoor D, Goodwin E, Channer KS, Jones TH. Testosterone replacement therapy improves insulin resistance, glycaemic control, visceral adiposity and hypercholesterolaemia in hypogonadal men with type 2 diabetes. Eur J Endocrinol. 2006;154:899-906.

4. Kenny AM, Prestwood KM, Gruman CA, Marcello KM, Raisz LG. Effects of transdermal testosterone on bone and muscle in older men with low bioavailable testosterone levels. J Gerontol A Biol Sci Med Sci. 2001;56:M266-72.

5. Calof OM, Singh AB, Lee ML, et al. Adverse events associated with testosterone replacement in middle-aged and older men: a meta-analysis of randomized, placebocontrolled trials. J Gerontol A Biol Sci Med Sci. 2005;60:1451-7.

6. Coviello AD, Kaplan B, Lakshman KM, Chen T, Singh AB, Bhasin S. Effects of graded doses of testosterone on erythropoiesis in healthy young and older men. The Journal of clinical endocrinology and metabolism. 2008;93:914-9.

7. Haddad RM, Kennedy CC, Caples SM, et al. Testosterone and cardiovascular risk in men: a systematic review and meta-analysis of randomized placebo-controlled trials. Mayo Clin Proc. 2007;82:29-39.

8. Kovac JR, Rajanahally S, Smith RP, Coward RM, Lamb DJ, Lipshultz LI. Patient satisfaction with testosterone replacement therapies: the reasons behind the choices. J Sex Med. 2014;11:553-62.

9. Dobs AS, Meikle AW, Arver S, Sanders SW, Caramelli KE, Mazer NA. Pharmacokinetics, efficacy, and safety of a permeation-enhanced testosterone transdermal system in comparison with bi-weekly injections of testosterone enanthate for the treatment of hypogonadal men. J Clin Endocrinol Metab. 1999;84:3469-78.

10. Shahidi NT. Androgens and erythropoiesis. N Engl J Med. 1973;289:72-80.

11. Palacios A, Campfield LA, McClure RD, Steiner B, Swerdloff RS. Effect of testosterone enanthate on hematopoiesis in normal men. Fertil Steril. 1983;40:100-4.

12. Bachman E, Feng R, Travison T, et al. Testosterone suppresses hepcidin in men: a potential mechanism for testosterone-induced erythrocytosis. J Clin Endocrinol Metab. 2010;95:4743-7.

13. Vigen R, O'Donnell CI, Baron AE, et al. Association of testosterone therapy with mortality, myocardial infarction, and stroke in men with low testosterone levels. JAMA. 2013;310:1829-36.

14. Masterson T, Molina M, Ibrahim E, Ramasamy R. Natesto Effects on Reproductive Hormones and Semen Parameters: Results from an Ongoing Single-center, Investigatorinitiated Phase IV Clinical Trial. Eur Urol Focus. 2018;4:333-5.

15. Rogol AD, Tkachenko N, Badorrek P, Hohlfeld JM, Bryson N. Phase 1 pharmacokinetics and phase 3 efficacy of testosterone nasal gel in subjects with seasonal allergies. Can Urol Assoc J. 2018;12:E349-E56. 
16. Rogol AD, Tkachenko N, Bryson N. Natesto, a novel testosterone nasal gel, normalizes androgen levels in hypogonadal men. Andrology. 2016;4:46-54.

17. MacIndoe JH, Perry PJ, Yates WR, Holman TL, Ellingrod VL, Scott SD. Testosterone suppression of the HPT axis. J Investig Med. 1997;45:441-7.

18. Bhasin S, Cunningham GR, Hayes FJ, et al. Testosterone therapy in men with androgen deficiency syndromes: an Endocrine Society clinical practice guideline. J Clin Endocrinol Metab. 2010;95:2536-59.

19. Hajjar RR, Kaiser FE, Morley JE. Outcomes of long-term testosterone replacement in older hypogonadal males: a retrospective analysis. J Clin Endocrinol Metab. 1997;82:3793-6.

20. Wang C, Swerdloff RS, Iranmanesh A, et al. Transdermal testosterone gel improves sexual function, mood, muscle strength, and body composition parameters in hypogonadal men. J Clin Endocrinol Metab. 2000;85:2839-53.

21. Ip FF, di Pierro I, Brown R, Cunningham I, Handelsman DJ, Liu PY. Trough serum testosterone predicts the development of polycythemia in hypogonadal men treated for up to 21 years with subcutaneous testosterone pellets. Eur J Endocrinol. 2010;162:385-90.

22. Ramasamy R, Masterson TA, Best JC, et al. Effect of Natesto on Reproductive Hormones, Semen Parameters and Hypogonadal Symptoms: A Single-Center, OpenLabel, Single-Arm Trial. J Urol. 2020:101097JU0000000000001078.

23. Gagnon DR, Zhang TJ, Brand FN, Kannel WB. Hematocrit and the risk of cardiovascular disease--the Framingham study: a 34-year follow-up. Am Heart J. 1994;127:674-82.

24. Fernandez-Balsells MM, Murad MH, Lane M, et al. Clinical review 1: Adverse effects of testosterone therapy in adult men: a systematic review and meta-analysis. J Clin Endocrinol Metab. 2010;95:2560-75.

\section{Figures and Tables}

Fig. 1. Followup hematocrit (Hct) levels during therapy with Natesto or testosterone cypionate 


\section{Polycythemia prevalence with intranasal vs. intramuscular testosterone}

(TC). Represents the number of patients reaching various Hct cutoffs during treatment with TC or Natesto.

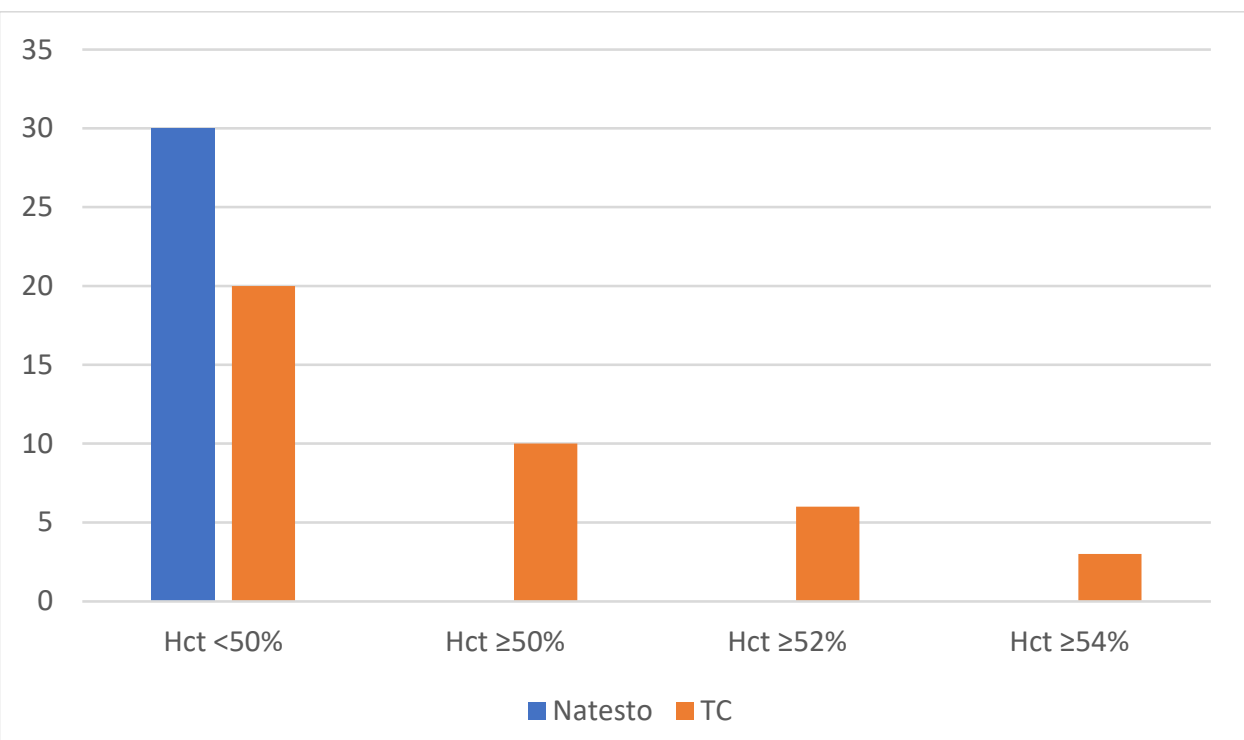

\begin{tabular}{|c|c|c|c|c|}
\hline & Overall & Natesto & TC & $\mathbf{p}$ \\
\hline Age & $45.3 \pm 13.2$ & $36.6 \pm 7.6$ & $54 \pm 11.8$ & $<0.001$ \\
\hline BMI & $29.7 \pm 5.8$ & $30.5 \pm 6.8$ & $28.6 \pm 4.6$ & 0.281 \\
\hline \multicolumn{5}{|l|}{ Smoking history } \\
\hline No & $46(76.7 \%)$ & $24(80 \%)$ & $22(73.3 \%)$ & \\
\hline Yes & $14(23.3 \%)$ & $6(20 \%)$ & $8(26.7 \%)$ & 0.542 \\
\hline \multicolumn{5}{|l|}{ Baseline } \\
\hline Testosterone & $244.5 \pm 101.4$ & $228.7 \pm 57.7$ & $260.4 \pm 130.6$ & 0.231 \\
\hline Hematocrit & $44.8 \pm 3.3$ & $44.8 \pm 2.2$ & $44.8 \pm 3.7$ & 0.994 \\
\hline \multicolumn{5}{|l|}{ Followup } \\
\hline Testosterone & $811.7 \pm 388.8$ & $640.3 \pm 302.4$ & $983.1 \pm 394.1$ & $<0.001$ \\
\hline Hematocrit & $46.5 \pm 3.6$ & $44.9 \pm 2.3$ & $48.1 \pm 3.9$ & $<0.001$ \\
\hline $\begin{array}{l}\text { Time followup } \\
\text { (months) }\end{array}$ & $5.7 \pm 3.4$ & $4.7 \pm 1.5$ & $6.9 \pm 4.3$ & 0.004 \\
\hline
\end{tabular}

Mean \pm standard deviation. BMI: body mass index; TC: testosterone cypionate.

\begin{tabular}{|c|c|c|c|c|c|c|c|c|c|c|}
\hline \multirow[t]{3}{*}{ Variable } & \multicolumn{5}{|c|}{ Univariable } & \multicolumn{5}{|c|}{ Multiple linear regression } \\
\hline & $\mathbf{R}^{2}$ & B & $95^{\circ}$ & CI & p & Adj. $\mathbf{R}^{2}$ & $\mathbf{B}$ & 950 & CI & p \\
\hline & & & Lower & Upper & & & & Lower & Upper & \\
\hline
\end{tabular}


Best et al

Polycythemia prevalence with intranasal vs. intramuscular testosterone

\begin{tabular}{|l|c|c|c|c|c|c|c|c|c|c|}
\hline Age in years & 0.080 & 0.08 & 0.01 & 0.14 & 0.029 & 0.134 & -0.01 & 0.08 & -0.10 & 0.796 \\
\hline Treatment group & & & & & & & & & & \\
\hline Natesto & & Ref. & & & & & Ref. & & & \\
\hline TC & 0.200 & 3.15 & 1.50 & 4.81 & $<0.001$ & & 3.24 & 0.74 & 5.73 & 0.012 \\
\hline BMI $\left(\mathrm{kg} / \mathrm{m}^{2}\right)$ & 0.0002 & -0.01 & -0.17 & 0.15 & 0.921 & & 0.04 & -0.12 & 0.19 & 0.644 \\
\hline Smoking history & & & & & & & & & & \\
\hline No & & Ref. & & & & & Ref. & & & \\
\hline Yes & 0.006 & 0.66 & -1.52 & 2.85 & 0.546 & & 0.42 & -1.69 & 2.53 & 0.694 \\
\hline $\begin{array}{l}\text { Followup } \\
\begin{array}{l}\text { tostosterone (10 } \\
\text { ng/dL increase) }\end{array}\end{array}$ & 0.050 & 0.02 & -0.003 & 0.05 & 0.085 & & 0.004 & -0.02 & 0.03 & 0.730 \\
\hline
\end{tabular}

B: unstandardized regression coefficient; BMI: body mass index; CI: confidence interval; $\mathrm{R}^{2}$ : coefficient of determination; adj. $\mathrm{R}^{2}$ : adjusted coefficient of determination; Ref.: reference value; TC: testosterone cypionate. 